\title{
17. Wahlverwandtschaften: Sind Freundschaften für die soziale Integration wichtiger geworden?
}

\author{
Anne Böger, Oliver Huxhold \& Julia K. Wolff
}

\section{Kernaussagen}

Im Jahr 2014 haben die 40- bis 85-Jährigen mehr Personen in ihrem engen Netzwerk als im Jahr 1996: Im Jahr 2014 nennen die Befragten im Durchschnitt etwa fünf Personen (4,9 Personen), die innen wichtig sind und mit denen sie regelmäßig Kontakt haben. Dies ist im Mittel etwa eine Person mehr als noch im Jahr 1996 (4,1 Personen).

Mehr Menschen haben im Jahr 2014 Freundinnen oder Freunde im engen Netzwerk als 1996 diese können auch von mehr Menschen um Rat oder Trost gefragt werden: Während im Jahr 1996 46,1 Prozent der Personen mindestens eine befreundete Person im engen Netzwerk hatten, sind es im Jahr 2014 schon 56,2 Prozent. Zudem hat mehr als ein Drittel (39,3 Prozent) der 40- bis 85-Jährigen im Jahr 2014 befreundete Personen, an die sie sich für einen Rat wenden können und 35,7 Prozent haben Freundinnen oder Freunde, die Trost spenden können. Im Jahr 1996 waren es noch 24,1 beziehungsweise 23,2 Prozent.

Altersunterschiede in der emotionalen Unterstützung durch Freundinnen oder Freunde sind im Jahr 2014 größer als noch im Jahr 1996: Bei Älteren ist der Zuwachs im Anteil von Personen mit Freundinnen und Freunden, die Trost spenden können, geringer ausgeprägt als im mittleren Alter. Dadurch haben sich Altersunterschiede zwischen 1996 und 2014 vergrößert. Weniger als ein Viertel (22,0 Prozent) der 70-bis 85-Jährigen nennt im Jahr 2014 Freundinnen oder Freunde, die emotionale Unterstützung leisten können. Bei den 40- bis 54-Jährigen sind es hingegen 44,8 Prozent.

\begin{abstract}
Altersunterschiede bei Freizeitaktivitäten mit Freundinnen oder Freunden werden zwischen 1996 und 2014 geringer: Im Jahr 2014 nennen mehr 40- bis 85-Jährige mindestens eine Freizeitaktivität mit Freundinnen und Freunden (61,6 Prozent) als noch im Jahr 1996 (50,9 Prozent). Bei den Älteren - vor allem bei den 66- bis 71-Jährigen - ist der Zuwachs stärker als bei Personen im mittleren Erwachsenenalter. Dadurch unterscheiden sich Ältere und Jüngere im Jahr 2014 weniger stark in den Freizeitaktivitäten mit Freundinnen und Freunden als noch im Jahr 1996.

Bildungsunterschiede bei Freizeitaktivitäten mit Freundinnen und Freunden nehmen zwischen 1996 und 2014 zu: Bei höher Gebildeten wächst der Anteil von Personen, die Freizeitaktivitäten mit befreundeten Personen berichten zwischen 1996 und 2014 - nicht so bei den niedrig Gebildeten, sodass sich Bildungsunterschiede vergrößert haben. Mehr als zwei Drittel (69,7 Prozent) der hoch Gebildeten berichten im Jahr 2014 Freizeitaktivitäten mit Freundinnen oder Freunden. Bei den niedrig Gebildeten ist es weniger als die Hälfte der Personen (43,9 Prozent).
\end{abstract}




\subsection{Einleitung}

Soziale Beziehungsnetzwerke sind eine zentrale Voraussetzung für Zufriedenheit, Sicherheit und persönliches Wachstum (Baumeister \& Leary 1995). Sie erfüllen nicht nur grundlegende Bedürfnisse nach Zugehörigkeit und Wertschätzung, sondern sind auch Quelle für Unterstützung, Wissen und neue Perspektiven sowie für positive Erlebnisse im Rahmen gemeinsamer Aktivitäten.

Im Zuge veränderter Lebensbedingungen und der Verbreitung postmaterialistischer Werte haben die Beziehungsnetzwerke der 40 - bis 85 -Jährigen in den letzten Jahrzehnten einen vielfältigen Wandel erfahren (vgl. Kapitel 13, 14, 15 und 16). Ein Zuwachs in der Erwerbsbeteiligung von Frauen, eine höhere geografische Mobilität sowie ein stärkeres Streben nach Selbstbestimmtheit und Selbstentfaltung haben die Ausgestaltung persönlicher Beziehungen beeinflusst. Personen im mittleren Erwachsenenalter leben seltener in langjährigen Ehen (vgl. Kapitel 13) und bleiben häufiger kinderlos (vgl. Kapitel 14). Durch eine sinkende Geburtenrate sind familiale Netzwerke häufig schmaler geworden. Zudem hat sich die Wohnentfernung zwischen Eltern und ihren erwachsenen Kindern vergrößert (vgl. Kapitel 14).

Vor dem Hintergrund dieser Entwicklungen wird nicht selten befürchtet, dass Personen in der zweiten Lebenshälfte immer weniger Bezugspersonen und Potenzial für soziale Unterstützung zur Verfügung haben. Denkbar ist jedoch auch, dass es zu einer Umgestaltung von Beziehungsnetzwerken und -modellen kommt, sodass Bedürfnisse nach Nähe und Unterstützung besser mit den veränderten Lebensbedingungen und Werthaltungen der 40 - bis 85 -Jährigen vereinbar sind. Ergebnisse des Deutschen Alterssurveys (DEAS) weisen darauf hin, dass eine hohe emotionale Nähe von Generationenbeziehungen auch über geografische Distanz aufrechterhalten werden kann (vgl. Kapitel 14; Engstler \& Huxhold 2010). Ebenso scheint ein Rückgang der Institution Ehe nicht zwangsweise mit einem Anstieg von Partnerlosigkeit, sondern eher mit einer stärkeren Verbreitung alternativer Partnerschaftsmodelle (zum Beispiel der nichtehelichen Partnerschaft) einherzuge- hen (vgl. Kapitel 13; Engstler \& Tesch-Roemer 2010). Ein Rückgang traditioneller Beziehungsstrukturen muss somit nicht zwangsläufig einen Verlust sozialer Integration bedeuten.

Auch gibt es Hinweise darauf, dass selbstgewählte Beziehungen für das Sicherstellen sozialer Integration an Bedeutung gewonnen haben. So zeigten frühere Studien und Befunde des DEAS, dass Freundinnen und Freunde für Personen in der zweiten Lebenshälfte zunehmend ein stabiler Bestandteil sozialer Netzwerke (Suanet, van Tilburg, \& van Groenou 2013) und eine bedeutsame Quelle für soziale Unterstützung sind (Huxhold, Mahne, \& Naumann 2010). Freundschaften können in besonderem Maße dazu geeignet sein, den Wunsch nach Nähe und Unterstützung mit einem zunehmend verbreiteten Individualisierungsstreben $\mathrm{zu}$ vereinen. Im Gegensatz zu familialen Beziehungen können außerfamiliale Netzwerke eigenständig, anhand eigener Interessen und Charakteristiken ausgewählt werden. Zudem sind Bindungen mit Freundinnen und Freunden weniger durch Gefühle der Verpflichtung und feste Normen gekennzeichnet als familiale Beziehungen. Insgesamt gewährleisten freundschaftliche Beziehungen somit ein hohes Potenzial für Selbstentfaltung, Selbstbestimmtheit sowie für den Austausch anregender Perspektiven und Informationen. Gleichzeitig können vor allem langjährige Freundinnen und Freunde auch eine wichtige Quelle für Nähe, Zugehörigkeitsempfinden und Unterstützung sein.

Angesichts dieser besonderen Potenziale kann vermutet werden, dass Freundinnen und Freunde innerhalb der letzten Jahrzehnte an Bedeutung gewonnen haben. Die Stärke des Bedeutungszuwachses könnte sich hierbei zwischen Alters- und Bildungsgruppen unterscheiden. Häufig wird die Veränderung beziehungsrelevanter Werthaltungen und Lebensbedingungen als Folge der Expansion von Bildung und Wohlstand im Verlaufe der Nachkriegszeit verstanden. Vor diesem Hintergrund sollte die wachsende Bedeutung von Freundschaften in der Gruppe der 40- bis 85-Jährigen vor allem bei späteren Geburtskohorten und hier verstärkt bei höher Gebildeten erkennbar sein. 
Generell ist davon auszugehen, dass sich sowohl die Wichtigkeit außerfamilialer Beziehungen als auch die Möglichkeiten solche Bindungen aufzubauen zwischen Personen unterschiedlichen Alters, Geschlechts und Bildungsniveaus unterscheiden. Aufgrund einer Zunahme funktionaler Einschränkungen mit dem Alter (vgl. Kapitel 8) kann sich das Bedürfnis nach sozialer Unterstützung erhöhen (Shaw, Krause, Liang, \& Bennett 2007). Dies kann zu einem Ungleichgewicht im Austausch von Unterstützungsleistungen zwischen den beteiligten Personen führen. In engen und vor allem familialen Beziehungen wird eine Verletzung der Reziprozitätsnorm für soziale Unterstützung aufgrund von stärkeren Verpflichtungsgefühlen eher akzeptiert als in weniger engen Freundschaften und Bekanntschaften (Ikkink \& van Tilburg 1998; Neyer, Wrzus, Wagner, \& Lang 2011). Denkbar ist somit, dass Familienbeziehungen bei einem einseitig erhöhten Unterstützungsbedarf leichter bewahrt werden können als außerfamiliale Beziehungen. Zudem leidet nicht nur die ältere Person selbst, sondern auch ihre gleichaltrigen Freundinnen und Freunde unter gesundheitlichen Verlusten, während familiale Netzwerke in der Regel unterschiedliche Generationen umfassen. Angesichts dieser Entwicklungen ist denkbar, dass Freundschaften im höheren Alter schlechter aufrechterhalten werden können als familiale Bindungen.

Der Stellenwert freundschaftlicher Beziehungen könnte sich auch zwischen den Geschlechtern unterscheiden. Studien weisen darauf hin, dass sich Männer für den Erhalt von emotionaler Unterstützung vor allem auf ihre Partnerin verlassen, während Frauen verstärkt außerfamiliale Beziehungen heranziehen (zum Beispiel Antonucci \& Akiyama 1987). Im Einklang mit dieser Idee zeigten frühere Befunde des DEAS, dass Frauen häufiger emotional enge Beziehungen zu Freundinnen und Freunden berichten als Männer (Huxhold et al. 2010). Keine Geschlechtsunterschiede zeigten sich hingegen in der Rolle von Freundinnen und Freunden als Quelle für Rat. Unterschiede zwischen Männern und Frauen sind somit vor allem in der emotio- nalen Enge von Freundschaften, weniger jedoch im Austausch von Rat und der Gestaltung gemeinsamer Aktivitäten zu erwarten.

Zuletzt könnte sich die Rolle von Freundschaften auch zwischen Bildungsgruppen unterscheiden. Studien weisen darauf hin, dass soziale Netzwerke von Personen mit niedriger Bildung häufiger familienzentriert sind als die von Personen mit höherer Bildung (van Groenou \& van Tilburg 2003). Bildungsunterschiede hinsichtlich berufsbedingter Mobilität sowie beruflichen Aufgaben könnten dazu beitragen, dass Personen mit höherer Bildung verstärkt außerfamiliale Kontakte knüpfen können beziehungsweise müssen und dadurch weniger auf die Familie zentriert sind (zum Beispiel Fors \& Lennartsson 2008). Des Weiteren beeinflussen finanzielle Ressourcen die Möglichkeiten für gemeinsame Freizeitaktivitäten, welche für die Entwicklung und Aufrechterhaltung außerfamilialer Beziehungen einen besonderen Stellenwert haben könnten. Auch Unterschiede in beziehungsrelevanten Werten könnten eine Rolle spielen. So gibt es Hinweise darauf, dass das Streben nach Individualität bei hoher Bildung und finanzieller Sicherheit stärker ausgeprägt ist als bei niedrigem Bildungsniveau (zum Beispiel Kroh 2008).

Insgesamt sind also sowohl ein Wandel in den sozialen Netzwerken und Aktivitäten über die letzten Jahre, als auch Unterschiede zwischen verschiedenen gesellschaftlichen Gruppen zu erwarten. Daher widmet sich dieses Kapitel den folgenden Fragestellungen:

1. Wie haben sich verschiedene Facetten sozialer Integration (enge Netzwerke, Potenzial für soziale Unterstützung, soziale Freizeitaktivitäten) gewandelt und wie unterscheiden sie sich zwischen gesellschaftlichen Gruppen?

2. Haben Freundschaften für die verschiedenen Facetten sozialer Integration (als Teil des engen Netzwerks, als Quelle für soziale Unterstützung, als Partner sozialer Freizeitaktivitäten) an Bedeutung gewonnen und wie unterscheidet sich ihre Bedeutung zwischen gesellschaftlichen Gruppen? 


\subsection{Datengrundlage ${ }^{1}$}

Daten. Für die Beantwortung der Fragestellungen werden die Daten der 40 - bis 85 -Jährigen Teilnehmerinnen und Teilnehmer des DEAS aus den Jahren 1996, 2002, 2008 und 2014 genutzt. Es werden vier Facetten sozialer Integration betrachtet: 1) das enge Netzwerk, 2) das Potenzial für informationelle Unterstützung, 3) das Potenzial für emotionale Unterstützung und 4) die sozialen Freizeitaktivitäten. Für alle Facetten ist hierbei vor allem der Anteil von Befragten mit Freundinnen und Freunden von Interesse. Angaben zu den vier Indikatoren sozialer Integration wurden in allen vier Befragungsjahren des DEAS von 1996 bis 2014 im Zuge des mündlichen Interviews erhoben.

Enges Netzwerk. Zur Erfassung des engen Netzwerks wurden die Befragten gebeten, bis zu acht Personen aufzuzählen, die ihnen wichtig sind und mit denen sie regelmäßig Kontakt haben. Anhand der Angaben wurde die Größe des engen Netzwerks (null bis acht) ermittelt. Zudem wurde der Anteil an Personen mit mindestens einem freundschaftlichen Kontakt (Freundin und Freund, Arbeitskollegin und Arbeitskollege, Nachbarin und Nachbar, Vereinsmitglied, Bekannte und Bekannter) in ihrem Netzwerk bestimmt.

Potenzial für informationelle Unterstützung. Zur Erfassung der Potenzials für informationelle Unterstützung wurden die Befragten gebeten bis zu fünf Personen zu nennen, an die sie sich wenden können, um nach Rat zu fragen. Aus den Angaben wurde die Anzahl potenzieller Ratgeberinnen und Ratgeber (null bis fünf) ermittelt. Des Weiteren wurde der Anteil an Personen mit zumindest einem freundschaftlichen Kontakt (Freundin und Freund, Arbeitskollegin und Arbeitskollege, Nachbarin und Nachbar, Vereinsmitglied, Bekannte und Bekannter) als Quelle für Rat bestimmt.

Potenzial für emotionale Unterstützung. Mit einem ähnlichen Maß wurde das Potenzial für

1 Die Daten des DEAS können für wissenschaftliche Zwecke kostenlos beim Forschungsdatenzentrum des DZA (www.fdz-dza.de) bezogen werden. emotionale Unterstützung abgebildet. Wiederum nannten die Befragten bis zu fünf Personen, an die sie sich wenden können, um Trost oder Aufmunterung zu erhalten. Neben der Gesamtanzahl von Personen, die Trost geben können (null bis fünf), wurde auch hier der Anteil an Personen mit mindestens einem freundschaftlichen Kontakt (Freundin und Freund, Arbeitskollegin und Arbeitskollege, Nachbarin und Nachbar, Vereinsmitglied, Bekannte und Bekannter) als Quelle für Trost ermittelt.

Soziale Freizeitaktivitäten. Die Freizeitgestaltung wurde über eine Auswahl verschiedener Aktivitäten innerhalb der letzten zwölf Monate erfasst (spazieren gehen, Sport treiben, künstlerisch tätig sein, kulturelle Veranstaltungen besuchen, Sportveranstaltungen besuchen, Gesellschaftsspiele spielen, Kurse und Vorträge besuchen). Für jede Aktivität gaben die Befragten an, ob sie diese innerhalb der letzten zwölf Monate meist allein oder mit anderen Personen (mit Partnerin und Partner, mit Verwandten, mit Freundinnen und Freunden, im Verein und mit Anderen) ausgeführt haben. Anhand der Antworten wurde die Summe der sozialen Aktivitäten (null bis sieben), das heißt aller Aktivitäten, die nicht meist alleine ausgeführt wurden, ermittelt. Zudem wurde der Anteil an Personen, die zumindest eine Freizeitaktivität mit befreundeten Personen (Aktivität mit Freundinnen und Freunden oder im Verein) ausgeführt haben, ermittelt.

Gruppierungsvariablen. Zur Prüfung von Altersunterschieden im Jahr 2014 wurden - analog zur Schichtung der Stichprobe - drei Altersgruppen verwendet: 40 bis 54 Jahre, 55 bis 69 Jahre und 70 bis 85 Jahre. Altersunterschiede im Wandel wurden anhand von Sechs-Jahres-Altersgruppen untersucht, um Überschneidungen zwischen Erhebungszeitpunkten und Altersgruppen zu vermeiden ( 42 bis 47 Jahre, 48 bis 53 Jahre, 54 bis 59 Jahre, 60 bis 65 Jahre, 66 bis 71 Jahre, 72 bis 77 Jahre, 78 bis 83 Jahre). Neben Altersunterschieden wurden auch Unterschiede nach Geschlecht und Bildung betrachtet. Zur Untersuchung von Bildungsunterschieden wur- 
den - basierend auf einer reduzierten ISCEDKlassifizierung - drei Bildungsgruppen unterschieden: Personen mit niedriger, mittlerer und hoher Bildung (vgl. Kapitel 2).

Analysen. In den Abbildungen und im Text sind gewichtete (gruppenspezifische) Mittelwerte oder Häufigkeiten angegeben. Um Gruppenunterschiede auf ihre statistische Signifikanz zu testen, wurden in Abhängigkeit vom Skalenniveau logistische Regressionen (dichotome Variablen) und Varianzanalysen (kontinuierliche Variablen) berechnet, wobei für die Stratifizierungsvariablen Altersgruppe, Geschlecht und Region (Ost-/Westdeutschland) kontrolliert wurde. Das genaue Vorgehen ist in Kapitel 2 beschrieben.

\subsection{Wandel und Unterschiede in der sozialen Integration}

Vergleicht man 2014 und 1996, zeigt sich, dass sich die hier betrachteten Facetten sozialer Integration bei den 40 - bis 85 -Jährigen eher positiv entwickelt haben. Allerdings differiert der Wandel zum Teil zwischen Bildungs- und Altersgruppen.

\section{Im Jahr 2014 haben die 40- bis 85-Jährigen mehr Personen in ihrem engen Netzwerk als im Jahr 1996.}

Die durchschnittliche Größe der engen Netzwerke hat sich von 1996 bis 2014 um fast eine Person erhöht (1996: 4,1 Personen, 2002: 4,7 Personen, 2008: 4,3 Personen, 2014: 4,9 Personen; vgl. Tabelle A 17-1 im Anhang). Dieser Trend ist für Personen unterschiedlichen Alters, Geschlechts und Bildungsniveaus gleichermaßen erkennbar. Allerdings bestehen im Jahr 2014 bedeutsame Unterschiede zwischen diesen Gruppen. Speziell berichten Personen im mittleren Erwachsenenalter und Frauen mehr Personen in ihrem Netzwerk als Ältere und Männer (vgl. Tabelle A 17-1 im Anhang). Des Weiteren verfügen Personen mit höherer Bildung über größere Netzwerke (5,2 Personen) als Personen mit mittlerer Bildung (4,8 Personen) und Niedriggebildete (4,4 Personen) (ohne Abbildung).

Die durchschnittliche Anzahl von Personen, die informationelle Unterstützung leisten können, hat sich von 1996 bis 2008 leicht reduziert (1996: 2,1 Personen, 2002: 2,0 Personen, 2008: 1,9 Personen; vgl. Tabelle A 17-2 im Anhang). Von 2008 bis 2014 kommt es hingegen in allen
Altersgruppen $\mathrm{zu}$ einem Zuwachs in der Anzahl potenzieller Ratgeberinnen und Ratgeber. In der Folge ist das durchschnittliche Potenzial für informationelle Unterstützung im Jahr 2014 (2,3 Personen) größer als 1996 (2,1 Personen). Allerdings ist der Langzeitzuwachs von 1996 bis 2014 nur bei den unter 72-Jährigen bedeutsam. Bei Personen, die 72 Jahre oder älter sind, ist die durchschnittliche Anzahl von Ratgeberinnen und Ratgebern im Jahr 2014 hingegen ähnlich hoch wie im Jahr 1996. Zudem unterscheidet sich der Anstieg des informationellen Unterstützungspotenzials zwischen den Geschlechtern. Speziell ist der Zuwachs von 1996 bis 2014 bei den Frauen etwas stärker als bei den Männern. Im Jahr 2014 berichten Personen im mittleren Erwachsenenalter und Frauen von mehr Potenzial für informationelle Unterstützung als Ältere und Männer (vgl. Tabelle A 17-2 im Anhang). Zudem ist die Anzahl von Ratgeberinnen und Ratgebern bei Hochgebildeten (2,5 Personen) höher als bei Personen mit mittlerem (2,2 Personen) und niedrigem Bildungsniveau (2,1 Personen) (ohne Abbildung).

Auch die durchschnittliche Anzahl von Personen, die emotionale Unterstützung leisten können, hat sich bis zum Jahr 2008 zunächst reduziert (1996: 2,0 Personen, 2002: 1,9 Personen, 2008: 1,8 Personen; vgl. Tabelle A 17-3 im Anhang). Von 2008 bis 2014 ist hingegen in fast allen Altersgruppen (außer bei den 78- bis 83-Jährigen) ein Zuwachs im Potenzial für emotionale Unterstützung erkennbar (Abbildung 17-1). Schaut man auf die Langezeittrends von 1996 bis 2014, zeigen sich bedeutsame Unter- 
schiede zwischen den Altersgruppen. Bei den unter 60-Jährigen ist die Anzahl von Personen, die emotionale Unterstützung leisten können im Jahr 2014 bedeutsam höher als im Jahr 1996. Bei den 60- bis 71-Jährigen gibt es hingegen keine bedeutsamen Veränderungen und bei den über 71-Jährigen ist die Anzahl von Personen, die Trost spenden können im Jahr 2014 sogar geringer als noch im Jahr 1996. Auch zwischen den Bildungsgruppen bestehen bedeutsame Unterschiede im Wandel. Vergleicht man 1996 und 2014 (ohne Abbildung), hat das Potenzial für emotionale Unterstützung bei den Hochgebildeten zugenommen (1996: 2,1 Personen, 2014: 2,3 Personen), während es sich bei den Niedriggebildeten reduziert hat (1996: 2,0 Personen, 2014:
1,7 Personen). Insgesamt bestehen im Jahr 2014 somit nicht nur größere Alters-, sondern auch größere Bildungsunterschiede im Potenzial für emotionale Unterstützung als noch im Jahr 1996.

Im Einklang mit dem altersdifferenziellen Wandel berichten Jüngere im Jahr 2014 bedeutsam mehr Personen, die Trost geben können, als Ältere (vgl. Tabelle A 17-3 im Anhang). Ebenso berichten Frauen von mehr Personen für emotionale Unterstützung als Männer (vgl. Tabelle A 17-3 im Anhang). Zudem bestehen bedeutsame Bildungsunterschiede: Hochgebildete haben hierbei mehr Personen, die Trost spenden können (2,3 Personen) als Personen mit mittlerem (2,0 Personen) und niedrigem Bildungsniveau (1,7 Personen) (ohne Abbildung).

Abbildung 17-1: Mittlere Anzahl von Personen, die Trost spenden können nach Alter, in den Jahren 1996, 2002, 2008 und 2014

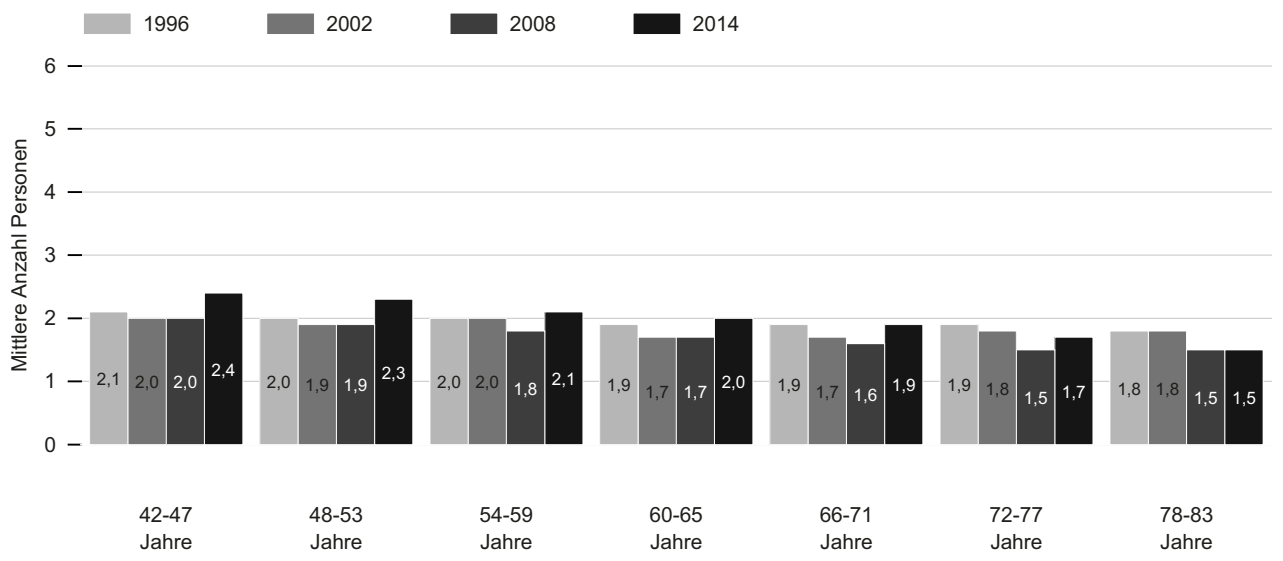

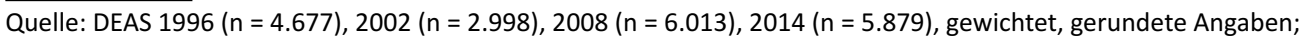
$(p<, 05)$.

Signifikante Abnahme für alle Altersgruppen ab 54 Jahren zwischen 1996 und 2008, signifikante Zunahme für alle außer den 78- bis 83-Jährigen zwischen 2008 und 2014. Signifikanter Unterschied zwischen 1996 und 2014 für alle außer die Altersgruppen 60 bis 65 Jahre und 66 bis 71 Jahre.

Die durchschnittliche Anzahl von Freizeitaktivitäten, die zusammen mit anderen Personen ausgeübt werden, hat sich von 1996 bis 2014 in allen Altersgruppen bedeutsam erhöht (1996: 2,4 Aktivitäten, 2002: 2,7 Aktivitäten, 2008: 2,9 Aktivitäten, 2014: 3,1 Aktivitäten; vgl. Tabelle A 17-4 im Anhang). In höheren Altersgruppen (vor allem bei den 66- bis 77-Jährigen) ist der Zuwachs hierbei stärker als bei den Jüngeren (Abbildung 17-2a). In der Folge sind Altersunterschiede in der Anzahl sozialer Freizeitaktivitäten im Jahr 2014 geringer als noch im Jahr 1996. Im Gegensatz dazu haben sich die Bildungsunterschiede in diesem Bereich vergrößert. Dies lässt sich darauf zurückführen, dass der Zuwachs sozialer Freizeitaktivitäten von 1996 bis 2014 bei den Niedriggebildeten geringer ausgeprägt ist als bei Personen mit höherer Bildung (Abbil- 
dung 17-2b). Im Jahr 2014 berichten jüngere Personen mehr soziale Freizeitaktivitäten als Ältere (vgl. Tabelle A 17-4 im Anhang). Zwischen Männern und Frauen unterscheidet sich die Anzahl sozialer Freizeitaktivitäten nicht bedeutsam (vgl. Tabelle A 17-4 im Anhang). Im Einklang mit den differenziellen Trends im Wandel berichten Personen mit höherer Bildung im Jahr 2014 mehr soziale Freizeitaktivitäten (3,6 Aktivitäten) als Personen mit mittlerem (3,0 Aktivitäten) und niedrigem Bildungsniveau (2,0 Aktivitäten) (ohne Abbildung).

Abbildung 17-2: Mittlere Anzahl von sozialen Freizeitaktivitäten nach Alter und Bildung, in den Jahren 1996, 2002, 2008 und 2014

a) Nach Alter

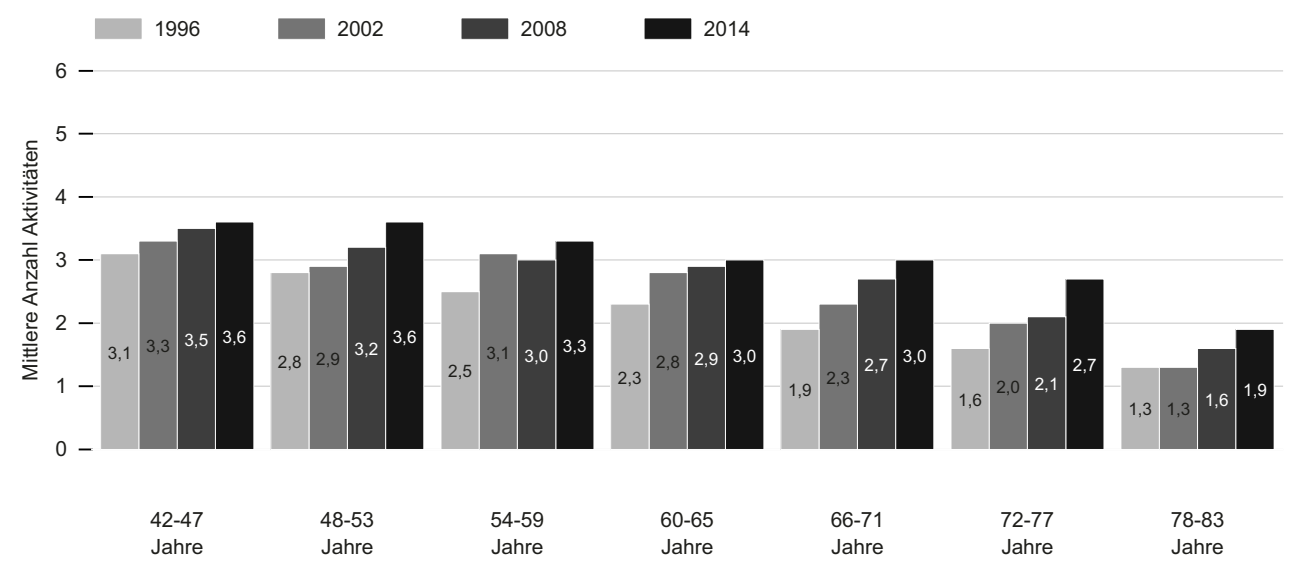

b) Nach Bildung

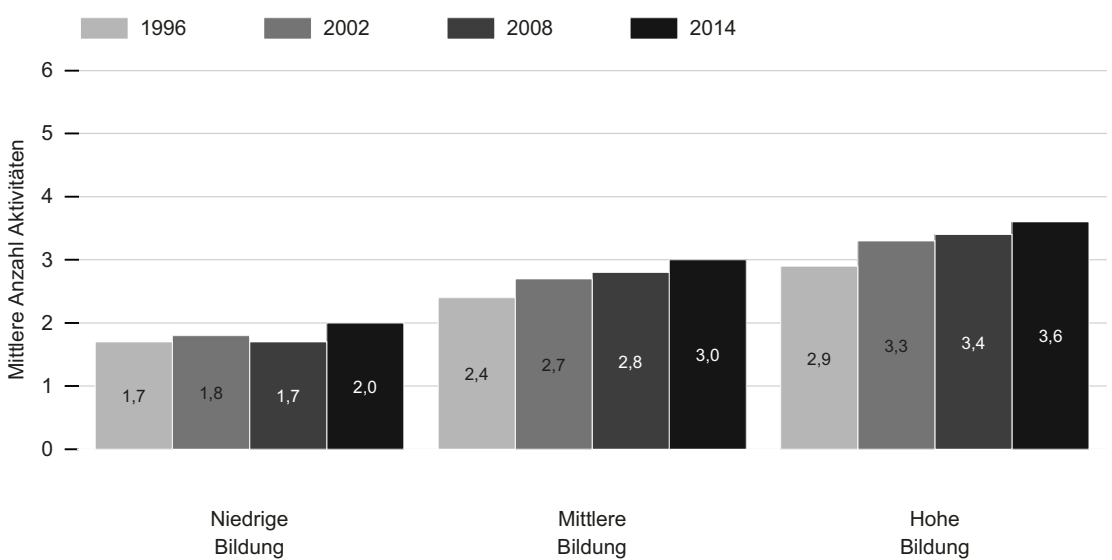

Quelle: DEAS $1996(n=4.550), 2002(n=2.883), 2008(n=6.190), 2014(n=5.984)$, gewichtet, gerundete Angaben; $(p<, 05)$.

a) Unterschiede zwischen 1996 und 2014 sind in allen Altersgruppen signifikant. In höheren Altergruppen (vor allem bei den 66- bis 77-Jährigen) ist der Anstieg signifikant stärker. b) Signifikante Zunahme zwischen 1996 und 2014 in allen Bildungsgruppen. Zunahme ist bei den höheren Bildungsgruppen stärker. 


\subsection{Wandel und Unterschiede in der Bedeutung von Freundschaften}

Nach der Betrachtung des Wandels verschiedener Facetten sozialer Integration stellt sich die wichtige Frage, inwiefern sich die beschriebenen Entwicklungen auf Veränderungen im Anteil von Personen mit Freundschaftsbeziehungen zurückführen lassen könnten.

\author{
Mehr Menschen haben im Jahr 2014 \\ Freundinnen oder Freunde im engen \\ Netzwerk als 1996 - diese können auch von \\ mehr Menschen um Rat oder Trost gefragt \\ werden.
}

Im Einklang mit den Erwartungen ist für alle der hier betrachteten Facetten sozialer Integration ein Zuwachs des Anteils von Personen mit Freundinnen und Freunden erkennbar (Abbildung 17-3). Erneut unterscheidet sich dieser Wandel jedoch zum Teil zwischen Alters- und Bildungsgruppen.

Abbildung 17-3: Anteile der Personen mit Freundinnen oder Freunden im engen Netzwerk, die Rat geben können, die Trost spenden können und die Partner für soziale Freizeitaktivitäten sind in den Jahren 1996, 2002, 2008 und 2014 (in Prozent)

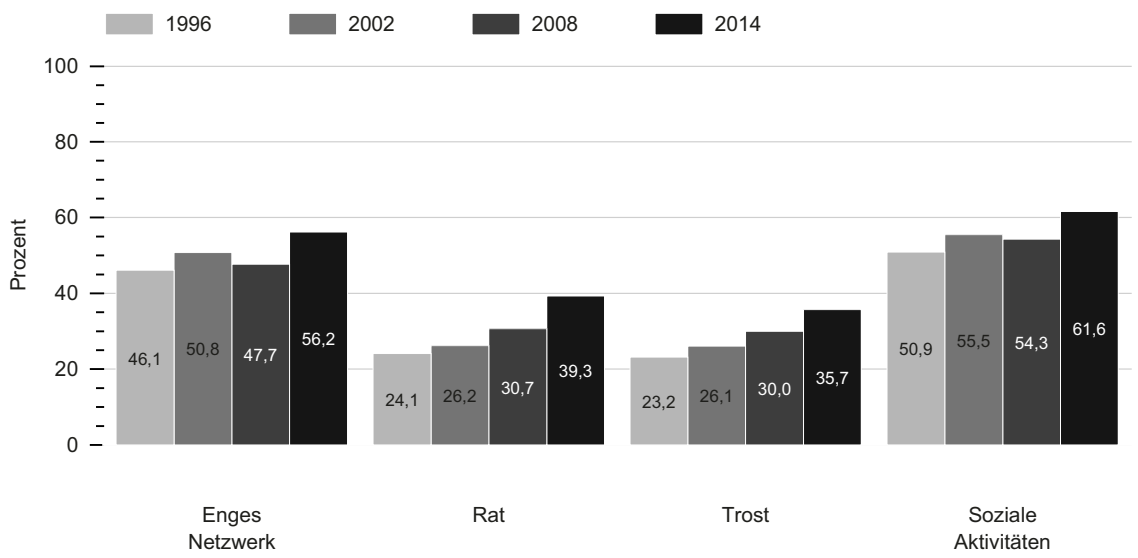

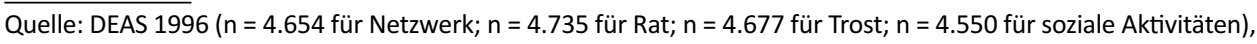
2002 ( $n=2.982$ für Netzwerk; $n=3.031$ für Rat; $n=2.998$ für Trost; $n=2.883$ für soziale Aktivitäten:), 2008 ( $n=6.098$ für Netzwerk; $n=6.055$ für Rat; $n=6.013$ für Trost; $n=6.190$ für soziale Aktivitäten), 2014 ( $n=5.940$ für Netzwerk; $\mathrm{n}=5.880$ für Rat; $\mathrm{n}=5.879$ für Trost; $\mathrm{n}=5.984$ für soziale Aktivitäten), gewichtet, gerundete Angaben; $(p<, 05)$. Für alle Indikatoren sind die Unterschiede zwischen 1996 und 2014 signifikant.

Während im Jahr 1996 nur 46,1 Prozent der 40- bis 85-Jährigen Freundinnen und Freunde in ihrem engen Netzwerk berichten, sind es im Jahr 2014 56,2 Prozent (Abbildung 17-3). Dieser Zuwachs ist für Personen unterschiedlichen Alters, Geschlechts und Bildungsniveaus gleichermaßen erkennbar. Wie in der Gesamtgröße des Netzwerks bestehen jedoch auch bei der Einbindung von Freudinnen und Freunden bedeutsame Unterschiede zwischen diesen Gruppen im Jahr 2014. Bei Älteren ist der Anteil von Personen mit Freundinnen und Freunden 
im engen Netzwerk geringer als bei Jüngeren (40-54 Jahre: 60,1 Prozent, 55-69 Jahre: 56,9 Prozent, 70-85 Jahre: 49,1 Prozent; vgl. Tabelle A 17-5 im Anhang). Zudem gibt es mehr Frauen als Männer, die Freundinnen und Freunde in ihrem engen Netzwerk berichten (Frauen: 59,7 Prozent, Männer: 52,5 Prozent; vgl. Tabelle A 17-5 im Anhang). Ebenso bestehen bedeutsame Unterschiede zwischen den Bildungsgruppen (ohne Abbildung): So nennen etwa 62,2 Prozent der Hochgebildeten, aber nur 54,1 Prozent der Personen mit mittlerer Bildung und 43,5 Prozent der Niedriggebildeten mindestens eine Freundin oder einen Freund als Teil ihres engen Netzwerks.

Auch im Bereich der sozialen Unterstützung finden sich Hinweise auf eine steigende Bedeutung von Freundschaften. Im Jahr 2014 berichten 39,3 Prozent der 40- bis 85-Jährigen von mindestens einer befreundeten Person, von der sie Ratschläge beziehen können. In 1996 war das nur bei rund einem Viertel (24,1 Prozent) der Personen der Fall (Abbildung 173). Wie beim Netzwerk, ist dieser Zuwachs bei Personen unterschiedlichen Alters, Geschlechts und Bildungsniveaus gleichermaßen erkennbar. Allerdings unterscheiden sich diese Gruppen im Vorkommen freundschaftlicher Ratgeberinnen und Ratgeber im Jahr 2014. Erneut ist der Anteil von Personen mit Freundinnen und Freunden, die Rat geben können, im höheren Alter geringer ausgeprägt (40-54 Jahre: 47,9 Prozent, 55-69 Jahre: 38,9 Prozent, 70-85 Jahre: 25,9 Prozent; vgl. Tabelle A 17-6 im Anhang). Zudem berichten mehr Frauen als Männer Freundinnen oder Freunde, die informationelle Unterstützung leisten können (Frauen: 42,0 Prozent, Männer: 36,3 Prozent; vgl. Tabelle A 17-6 im Anhang). Zudem bestehen deutliche Unterschiede zwischen den Bildungsgruppen. So nennen rund 46,3 Prozent der Personen mit höherer Bildung freundschaftliche Kontakte als Quelle für Rat, während es in der Gruppe der Personen mit mittlerer Bildung 36,6 Prozent sind und bei den Niedriggebildeten nur etwa ein Viertel (25,1 Prozent) der Personen ist (ohne Abbildung).

\section{Altersunterschiede in der emotionalen Unterstützung durch Freundinnen oder Freunde sind im Jahr 2014 größer als noch im Jahr 1996.}

Wie die Entwicklung des Gesamtpotenzials für emotionale Unterstützung (Abbildung 17-1), unterscheidet sich der Wandel im Vorkommen emotionaler Unterstützung durch Freundinnen und Freunde zwischen Personen unterschiedlichen Alters. Insgesamt ist der Anteil von Personen mit Freundinnen und Freunden, die Trost geben können, im Jahr 2014 größer als im Jahr 1996 (1996: 23,2 Prozent, 2014: 35,7 Prozent; Abbildung 17-3). Allerdings ist die Stärke des Zuwachses bei den Älteren deutlich geringer ausgeprägt (Abbildung 17-4). Die zuvor berichtete Vergrößerung von Altersunterschieden im Potenzial für emotionale Unterstützung (Abbildung 17-1) scheint somit auch auf eine geringere Miteinbindung freundschaftlicher Unterstützer bei Älteren zurückführbar zu sein. Im Gegensatz dazu ist der Zuwachs im Vorkommen von Trost durch Freundinnen und Freunde in allen Bildungsgruppen ähnlich stark ausgeprägt. Es finden sich also keine Hinweise darauf, dass die Bildungsunterschiede im Wandel des emotionalen Unterstützungspotenzials auf einen unterschiedlich starken Zuwachs in der Einbeziehung befreundeter Personen zurückführbar sind.

Im Jahr 2014 nennt weniger als ein Viertel (22,0 Prozent) der 70-bis 85-Jährigen Freundinnen und Freunde, von denen sie Trost erhalten können. Bei den 55- bis 69-Jährigen sind es hingegen 34,8 Prozent und bei den 40- bis 54-Jährigen sogar 44,8 Prozent (vgl. Tabelle A 17-7 im Anhang). Zudem bestehen deutliche Geschlechtsunterschiede. Während 45,4 Prozent der Frauen mindestens eine Freundin oder einen Freund haben, an die oder den sie sich wenden können, um emotional unterstützt zu werden, ist es bei den Männern nur ein Viertel der Personen (25,2 Prozent; vgl. Tabelle A 17-7 im Anhang). Erneut gibt es auch bedeutsame Unterschiede zwischen den Bildungsgruppen (ohne Abbildung). Während in der Gruppe der Niedriggebildeten weniger als ein Viertel der Personen (22,3 Prozent) Freundinnen und Freunde, die Trost spenden können berichtet, sind es in der Gruppe der Personen mit mittlerer Bildung 33,9 Prozent und bei den Hochgebildeten 41,3 Prozent. 
Abbildung 17-4: Anteile der Personen mit Freundinnen und Freunden, die Trost spenden können nach Alter, in den Jahren 1996, 2002, 2008 und 2014 (in Prozent)

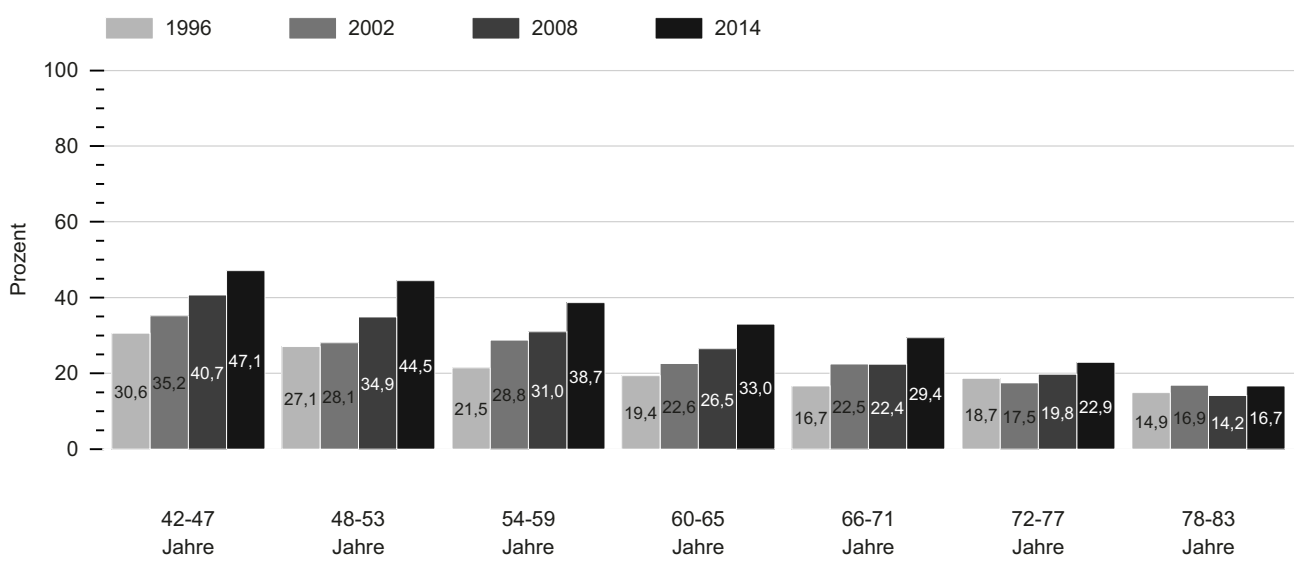

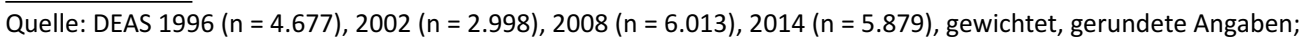
$(p<, 05)$.

Signifikante Zunahme von 1996 bis 2014 in allen Altersgruppen außer bei den 78- bis 83-Jährigen. Stärke des Zuwachses ist in älteren Altersgruppen signifikant kleiner.

\section{Altersunterschiede bei Freizeitaktivitäten mit Freundinnen oder Freunden werden zwischen 1996 und 2014 geringer.}

Im Jahr 1996 hat nur circa die Hälfte (50,9 Prozent) der 40 - bis 85 -Jährigen mindestens eine Freizeitaktivität mit Freundinnen und Freunden angegeben. Im Jahr 2014 sind es schon fast zwei Drittel (61,6 Prozent; Abbildung 17-3). Wie bei der Gesamtanzahl sozialer Freizeitaktivitäten ist dieser Wandel bei älteren Personen (vor allem bei den 66- bis 71-Jährigen) stärker ausgeprägt als bei den Jüngeren (Abbildung 17-5a). In der Folge haben sich Altersunterschiede im Auftreten von Freizeitaktivitäten mit befreundeten Personen von 1996 zu 2014 verringert. Die wachsende Anzahl an sozialen Freizeitaktivitäten bei Älteren (Abbildung 17-2) geht möglicherweise mit verbesserten Chancen für den Aufbau freundschaftlicher Beziehungen einher. Ebenso könnte eine positive Gesundheitsentwicklung bei Älteren und ihren gleichaltrigen Freundinnen und Freunden (vgl. Kapitel 8) dazu führen, dass gemeinsame Freizeitaktivitäten länger aufrechterhalten werden.

\section{Bildungsunterschiede bei Freizeitaktivitäten mit Freundinnen und Freunden nehmen zwischen 1996 und 2014 zu.}

Wie die Bildungsunterschiede in der Anzahl sozialer Freizeitaktivitäten (Abbildung 17-2), haben auch Bildungsunterschiede im Vorkommen von Freizeitaktivitäten mit Freundinnen und Freunden seit 1996 zugenommen (Abbildung 17-5b). Speziell ist es nur bei den Personen mit mittlerer und hoher Bildung, nicht aber bei Personen mit niedriger Bildung zu einem bedeutsamen Anstieg im Anteil von Personen, die Freizeitaktivitäten mit befreundeten Personen berichten, gekommen. Möglicherweise geht der bildungsdifferenzielle Wandel in der Anzahl sozialer Freizeitaktivitäten (Abbildung 17-2) mit einer ungleichen Entwicklung von Chancen für den Aufbau von Freundschaften einher. Auch könnten sich Möglichkeiten zur außerfamilialen Vernetzung, vor allem im beruflichen Bereich, bildungsdifferenziell gewandelt haben. Die Anzahl von Partnerinnen und Partnern für die Durchführung gemeinsamer Freizeitaktivitäten könnte sich daher unter Personen mit niedriger Bildung weniger günstig entwickelt haben als unter Personen mit höherer Bildung. 


\section{Abbildung 17-5: Anteile der Personen, die mindestens eine Freizeitaktivität mit Freundinnen und Freunden ausführen nach Alter und Bildung, in den Jahren 1996, 2002, 2008 und 2014 (in Prozent)}

a) Nach Alter

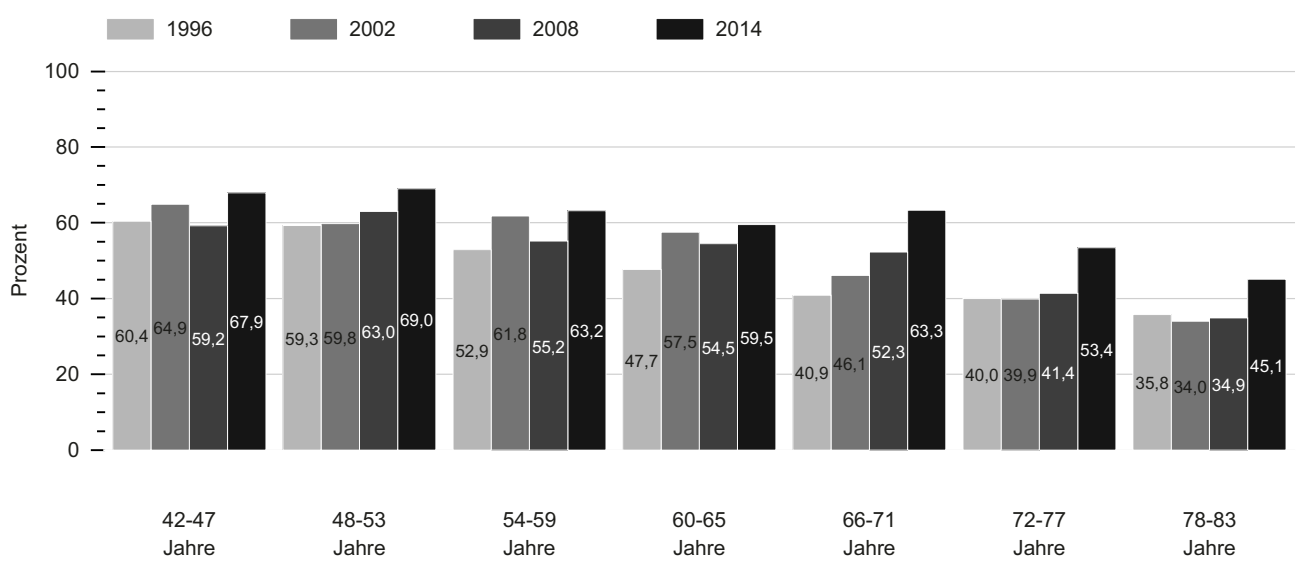

b) Nach Bildung

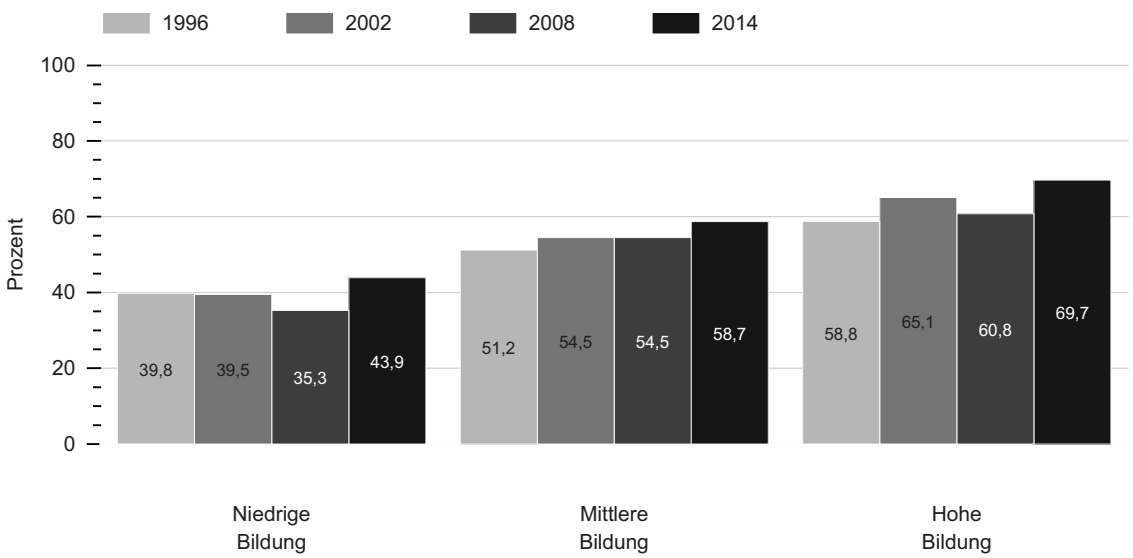

Quelle: DEAS $1996(n=4.550), 2002(n=2.883), 2008(n=6.190), 2014(n=5.984)$, gewichtet, gerundete Angaben; $(p<, 05)$. a) Unterschiede zwischen 1996 und 2014 sind in allen Altersgruppen signifikant. In höheren Altergruppen ist der Anstieg signifikant stärker. b) Bei niedrig Gebildeten besteht kein signifikanter Unterschied zwischen 1996 und 2014. Unterschied bei mittlerer und hoher Bildung ist signifikant.

Im Jahr 2014 werden Freizeitaktivitäten mit befreundeten Personen von Jüngeren häufiger berichtet als von Älteren (40 bis 54 Jahre: 67,7 Prozent, 55 bis 69 Jahre: 61,6 Prozent, 70 bis 85 Jahre: 51,8 Prozent; vgl. Tabelle A 17-8 im Anhang). Zudem gibt es mehr Frauen als Männer, die mindestens eine Freizeitaktivität mit Freundinnen oder Freunden ausüben (Frau: 62,7 Prozent, Männer: 60,4 Prozent; vgl. Tabelle A 17-8 im Anhang). Im Einklang mit den differenziellen Trends im Wandel, zeigen sich zudem bedeutsame Unterschiede zwischen den Bildungsgruppen. Während in der Gruppe der Hochgebildeten mehr als zwei Drittel (69,7 Prozent; Abbildung 17-5b) der Personen Freizeitaktivitäten mit Freundinnen und Freunden berichten, sind es bei den Personen mit mittlerem Bildungsniveau 58,7 Prozent und bei Personen mit niedrigem Bildungsniveau nur 43,9 Prozent. 


\subsection{Diskussion und Implikationen}

Soziale Beziehungen gelten als wichtige Ressource für positive Emotionen und Zufriedenheit sowie Gesundheit (zum Beispiel Berkman, Glass, Brissette, \& Seeman 2000). Sie erfüllen nicht nur Bedürfnisse nach Zugehörigkeit und Unterstützung (Baumeister \& Leary 1995), sondern fördern auch positive Erfahrungen im Rahmen gemeinsamer Aktivitäten.

Angesichts dieser Relevanz ist es erfreulich, dass sich viele Aspekte der sozialen Integration der 40- bis 85-Jährigen von 1996 bis 2014 positiv entwickelt haben. Zumindest teilweise scheint der Zuwachs sozialer Integration hierbei auf eine stärkere Einbindung freundschaftlicher Beziehungen zurückzuführen zu sein. Immer häufiger nennen Personen in der zweiten Lebenshälfte Freundinnen und Freunde als Teil ihres engen Netzwerkes, als Quelle für soziale Unterstützung und als Partner für Freizeitaktivitäten. Denkbar ist, dass der Bedeutungszuwachs von Freundschaften eine Anpassung an veränderte Lebensbedingungen, Werte und Normen repräsentiert (Inglehart 2008; Suanet et al. 2013). Vor dem Hintergrund sich intensivierender Wünsche nach Selbstverwirklichung und Selbstbestimmtheit könnten Freundschaften an Relevanz gewonnen haben, da sie stärker als familiale Beziehungen anhand eigener Interessen und Charakteristiken ausgewählt werden können. Häufig sind außerfamiliale Bindungen auch weniger durch moralische Verpflichtung und verbindliche Normen gekennzeichnet als familiale Beziehungen.

Trotz ihres weniger verpflichtenden Charakters haben Freundinnen und Freunde nicht nur als Partner für Freizeitaktivitäten sondern auch als enge Bezugsperson und Quelle für Unterstützung an Relevanz gewonnen. Nur bei den über 70-Jährigen ist dieser Trend derzeit noch weniger stark erkennbar. $\mathrm{Zu}$ vermuten ist, dass die geringere Einbindung von Freundschaften dazu beigetragen hat, dass sich das Potenzial für emotionale Unterstützung bei den Ältesten von 1996 bis 2014 leicht reduziert hat. Die Altersunterschiede in der Entwicklung freundschaftlicher Bezugspersonen könnten auf Unterschiede im Wandel von Werten und Lebensbedingungen zurückzuführen sein. Es ist davon auszugehen, dass frühere Geburtskohorten vom Wandel beziehungsrelevanter Werthaltungen und Normen weniger geprägt wurden als spätere. Im Gegensatz zu jüngeren Geburtsjahrgängen sind die älteren Kohorten derzeit auch noch kaum von einer Verschmälerung familialer Netzwerke durch vermehrte Kinderlosigkeit betroffen (vgl. Kapitel 14). Dies könnte dazu beitragen, dass der Bedeutungszuwachs von Freundschaften in älteren Kohorten abgeschwächt verläuft und bislang nur für einige Aspekte sozialer Integration erkennbar ist. Gerade für die Erfüllung intimer Unterstützungsleistungen - wie der Unterstützung durch Trost und Aufmunterung - können höhere Barrieren für den Einbezug nichtfamilialer Beziehungen bestehen. Hinzu kommt, dass Netzwerke im höheren Alter, insbesondere Freundschaften mit Gleichaltrigen oder Älteren, stärker von sozialen Verlusten und gesundheitlichen Einschränkungen betroffen sind als die von Personen im mittleren Erwachsenenalter. Gerade für den Austausch von Trost, für den in der Regel nur wenige, enge Freundschaften relevant sind, können diese altersbedingten Verluste besonders folgenreich und schwer zu kompensieren sein. Im Einklang mit dieser Vermutung bestehen im Jahr 2014 deutliche Altersunterschiede in der emotionalen Unterstützung durch Freundinnen und Freunde. In anderen Bereichen hingegen, scheint die soziale Integration der Älteren zunehmend von einer verbesserten Gesundheit und steigenden Lebenserwartung profitiert $\mathrm{zu}$ haben. So hat das Ausmaß sozialer Freizeitaktivitäten, auch solcher, die im Freundeskreis durchgeführt werden, bei den Älteren besonders stark zugenommen. Diese Entwicklung erscheint besonders positiv, wenn man bedenkt, dass soziale Aktivitäten mit Freundinnen und Freunden gerade bei Personen im Ruhestandsalter die Entwicklung des Wohlbefindens positiv beeinflussen können (Huxhold, Miche, \& Schüz 2014). Denkbar ist, dass die positive Entwicklung freizeitlicher Aktivitäten Älterer durch politische Maßnahmen 
zur Steigerung aktiven und engagierten Alterns gefördert wurde (vgl. Kapitel 5). Zudem könnte ein Zusammenhang zum häufigeren Ausüben von sportlicher Aktivität im Alter bestehen (vgl. Kapitel 9).

Im Gegensatz zu Altersunterschieden haben Bildungsunterschiede beim Ausüben von Freizeitaktivitäten mit befreundeten Personen seit 1996 leicht zugenommen. Im Jahr 2014 berichten Niedriggebildete seltener von Freundinnen und Freunden als Aktivitätspartner und Bezugsperson und sie sind auch insgesamt weniger gut integriert. Denkbar ist, dass sich finanzielle und gesundheitliche Ressourcen, welche vor allem für die Durchführung sozialer Freizeitaktivitäten relevant sind, bei Personen mit höherer Bildung günstiger entwickelt haben als bei Niedriggebildeten. So haben Bildungsunterschiede in der funktionalen Gesundheit zugenommen (vgl. Kapitel 8). Ebenso ist vorstellbar, dass eine lockere Vernetzung mit außerfamilialen Beziehungen in der Ausbildung und beruflichen Entwicklung höher Gebildeter besonders stark an Relevanz gewonnen hat. Insgesamt könnte es für Personen mit hoher Bildung in verschiedensten Bereichen sozialer Integration nicht nur einfacher, sondern auch wichtiger sein, außerfamiliale Beziehungen aufzubauen.

Im Gegensatz dazu scheinen Geschlechtsunterschiede hinsichtlich Ressourcen, Zielen und Bedürfnissen vor allem auf die Ausgestaltung emotional enger Beziehungsnetzwerke zu wirken. Im Jahr 2014 nennen Männer seltener Freundinnen und Freunde, die Trost spenden können, und auch insgesamt weniger Bezugspersonen und Unterstützungspotenzial. Auch die Kontakthäufigkeit und emotionale Enge in der Beziehung zu den eigenen Kindern ist bei Männern geringer ausgeprägt (vgl. Kapitel 14). Denkbar ist, dass die Geschlechtsunterschiede bei der sozialen Integration mit Unterschieden bei den sozialrelevanten Normen und Zielen in Verbindung stehen. Es wird vermutet, dass der Aufbau sozialer Kompetenz und das Zulassen emotionaler Expressivität (vor allem von negativen Gefühlen, vgl. Kapitel 11) in der Sozialisation von Frauen stärker gefördert werden als in der von Männern. Möglicherweise ist es für Frauen somit nicht nur einfacher, sondern es ist ihnen auch wichtiger, emotional enge Beziehungen aufzubauen. Männer hingegen scheinen sich für die Erfüllung intimer Bedürfnisse wie Trost vor allem auf die (Ehe-)Partnerin zu verlassen (Antonucci \& Akiyama 1987).

Angesichts der Unterschiede bei der sozialen Einbindung könnte vermutet werden, dass Ältere, Männer und Niedriggebildete ein höheres Risiko haben, sich unzureichend integriert zu fühlen. Tatsächlich berichten Personen mit niedriger Bildung häufiger Gefühle der Einsamkeit (vgl. Kapitel 18). Auch ein Mangel an finanziellen Ressourcen, den Niedriggebildete häufig erleben (vgl. Kapitel 6), erhöht das Risiko, sich ausgeschlossen zu fühlen (vgl. Kapitel 18). Ältere Personen und Männer hingegen scheinen sich trotz ihrer tendenziell schlechteren Integration in persönliche Beziehungsnetzwerke nicht häufiger einsam zu fühlen als jüngere Personen und Frauen (vgl. Kapitel 18). Es kann somit vermutet werden, dass die berichteten Unterschiede bei den sozialen Netzwerken und Aktivitäten nicht immer ein Defizit repräsentieren, sondern auch Ausdruck unterschiedlicher Bedürfnisse und Ziele sein können. Wie zuvor diskutiert, könnte das Ausmaß persönlicher und vor allem emotional enger Beziehungen für Frauen wichtiger sein als für Männer. In ähnlicher Weise wird vermutet, dass für Ältere vor allem die emotionale Qualität, weniger hingegen die Anzahl sozialer Beziehungen, von Bedeutung ist (Carstensen, Isaacowitz, \& Charles 1999).

Dennoch können Unterschiede bei der Größe und Vielfalt sozialer Netzwerke mit unterschiedlichen Risiken und Potenzialen einhergehen. Für den Austausch von Informationen, welche beispielsweise die Weiterentwicklung beruflicher Lebenspläne fördern können, sind breitgefächerte Netzwerke aus Freundinnen und Freunden, Bekannten sowie Kolleginnen und Kollegen häufig von besonderer Relevanz (Lin \& Dumin 1986; Yakubovich 2005). Zudem können negative Veränderungen sozialer Ressourcen bei Personen mit kleinen Netzwerken eher zu negativen Konsequenzen wie Einsamkeit führen als bei Personen, die umfassend integriert sind (Böger \& Huxhold 2016). Auch haben Personen mit stark familienzentrierten Netzwerken ein höheres Depressionsrisiko als solche, die vielfäl- 
tigere Beziehungen berichten (Fiori, Antonucci, \& Cortina 2006).

Insgesamt können große und vielfältige Beziehungsnetzwerke eine Ressource für die persönliche Entwicklung und die Bewältigung kritischer Ereignisse sein. Zudem könnte die Vielfalt sozialer Netzwerke - im Sinne einer Kombination ausreichender familialer und freundschaftlicher Integration - für die soziale Zufriedenheit Älterer immer mehr an Bedeutung gewinnen. Sowohl der Verlust von Freundinnen und Freunden sowie problematische Bedingungen zur Entwicklung außerfamilialer Beziehungen sollten als Risikofaktoren für die Lebensqualität von Personen in der zweiten Lebenshälfte stärker berücksichtigt werden. Zudem kann eine Förderung von Aktivitäten

\section{Literatur}

Antonucci, T. C., \& Akiyama, H. (1987). An examination of sex differences in social support among older men and women. Sex roles, 17(11-12), 737-749. doi: 10.1007/BF00287685.

Baumeister, R. F., \& Leary, M. R. (1995). The need to belong: desire for interpersonal attachments as a fundamental human motivation. Psychological Bulletin, 117(3), 497-529. doi: 10.1037/0033-2909.117.3.497.

Berkman, L. F., Glass, T., Brissette, I., \& Seeman, T. E. (2000). From social integration to health: Durkheim in the new millennium. Social Science \& Medicine, 51(6), 843-857. doi: 10.1016/s0277-9536(00)00065-4.

Böger, A., \& Huxhold, O. (2016). Do antecedents and consequences of loneliness change across the second half of life? (eingereichtes Manuskript).

Carstensen, L. L., Isaacowitz, D. M., \& Charles, S. T. (1999). Taking time seriously - A theory of socioemotional selectivity. American Psychologist, 54(3), 165181. doi: 10.1037//0003-066x.54.3.165.

Engstler, H., \& Huxhold, O. (2010). Beeinflusst die Beziehung älterer Menschen zu ihren erwachsenen Kindern die räumliche Nähe zwischen den Generationen? Wechselbeziehungen zwischen Wohnentfernung, Kontakthäufigkeit und Beziehungsenge im Längsschnitt. In: A. Ette, K. Ruckdeschel \& R. Unger (Hrsg.) Potenziale intergenerationaler Beziehungen: Chancen und Herausforderungen für die Gestaltung des demografischen Wandels (S. 175-197). Würzburg: Ergon. und Begegnungsstätten, welche einen Aufbau freundschaftlicher Beziehungen ermöglichen, ein wertvoller Ansatzpunkt für Interventionen zur Verbesserung der Zugehörigkeit und Lebensqualität im Alter sein. Angesichts der zentralen Funktionen sozialer Beziehungen, sollte auch künftig untersucht werden, wie sich die persönlichen Netzwerke der 40 - bis 85 -Jährigen im sozialen Wandel entwickeln. Insbesondere Trends in der sozialen Einbindung von Menschen mit geringer Bildung und der ältesten Mitbürgerinnen und Mitbürgern müssen weiter erforscht werden. Sollte sich die Ungleichheit von sozialen Aktivitäts- beziehungsweise Unterstützungsmöglichkeiten verschärfen, könnten Maßnahmen zur Förderung der sozialen Integration ratsam sein.

Engstler, H., \& Tesch-Roemer, C. (2010). Lebensformen und Partnerschaft. In: A. Motel-Klingebiel, S. Wurm \& C. Tesch-Roemer (Hrsg.) Altern im Wandel: Befunde des Deutschen Alterssurveys (DEAS) (S. 163-187). Stuttgart: Kohlhammer.

Fiori, K. L., Antonucci, T. C., \& Cortina, K. S. (2006). Social network typologies and mental health among older adults. The Journals of Gerontology Series B: Psychological Sciences and Social Sciences, 61(1), P25-P32. doi: 10.1093/geronb/61.1.P25.

Fors, S., \& Lennartsson, C. (2008). Social mobility, geographical proximity and intergenerational family contact in Sweden. Ageing and Society, 28(02), 253-270. doi: $10.1017 /$ S0144686X07006617.

Groenou, M. I. B. van, \& Tilburg, T. van (2003). Network size and support in old age: differentials by socio-economic status in childhood and adulthood. Ageing and Society, 23, 625-645. doi: 10.1017/ s0144686x0300134x.

Huxhold, O., Mahne, K., \& Naumann, D. (2010). Soziale Integration. In: A. Motel-Klingebiel, S. Wurm \& C. Tesch-Roemer (Hrsg.) Altern im Wandel. Befunde des Deutschen Alterssurveys (DEAS). (S. 215-233). Stuttgart: Kohlhammer.

Huxhold, O., Miche, M., \& Schüz, B. (2014). Benefits of having friends in older ages: Differential effects of informal social activities on well-being in middle-aged and older adults. The Journals of Gerontology - Series 
B: Psychological Sciences and Social Sciences, 69(3), 366-375. doi: 10.1093/geronb/gbt029.

Ikkink, K. K., \& Tilburg, T. van (1998). Do older adults' network members continue to provide instrumental support in unbalanced relationships? Journal of Social and Personal Relationships, 15(1), 59-75. doi: 10.1177/0265407598151004.

Inglehart, R. F. (2008). Changing values among western publics from 1970 to 2006. West European Politics, 31(1-2), 130-146. doi: 10.1080/01402380701834747.

Kroh, M. (2008). Wertewandel: Immer mehr Ost-und Westdeutsche ticken postmaterialistisch. DIW Wochenbericht, 75(34), 480-486.

Lin, N., \& Dumin, M. (1986). Access to occupations through social ties. Social networks, 8(4), 365-385. doi: 10.1016/0378-8733(86)90003-1
Neyer, F. J., Wrzus, C., Wagner, J., \& Lang, F. R. (2011). Principles of Relationship Differentiation. European Psychologist, 16(4), 267-277. doi: 10.1027/1016-9040/ a000055.

Shaw, B. A., Krause, N., Liang, J., \& Bennett, J. (2007). Tracking changes in social relations throughout late life. The Journals of Gerontology Series B: Psychological Sciences and Social Sciences, 62(2), S90-S99. doi: 10.1093/geronb/62.2.590.

Suanet, B., Tilburg, T. G. van, \& Groenou, M. I. B. van (2013). Nonkin in Older Adults' Personal Networks: More Important Among Later Cohorts? The Journals of Gerontology Series B: Psychological Sciences and Social Sciences, 68(4), 633-643. doi: 10.1093/geronb/gbt043.

Yakubovich, V. (2005). Weak ties, information, and influence: How workers find jobs in a local Russian labor market. American sociological review, 70(3), 408-421. doi: $10.1177 / 000312240507000303$.

Open Access Dieses Kapitel wird unter der Creative Commons Namensnennung 2.5 International Lizenz (http://creativecommons.org/licenses/by/2.5/deed.de) veröffentlicht, welche die Nutzung, Vervielfältigung, Bearbeitung, Verbreitung und Wiedergabe in jeglichem Medium und Format erlaubt, sofern Sie den/die ursprünglichen Autor(en) und die Quelle ordnungsgemäß nennen, einen Link zur Creative Commons Lizenz beifügen und angeben, ob Änderungen vorgenommen wurden.

Die in diesem Kapitel enthaltenen Bilder und sonstiges Drittmaterial unterliegen ebenfalls der genannten Creative Commons Lizenz, sofern sich aus der Abbildungslegende nichts anderes ergibt. Sofern das betreffende Material nicht unter der genannten Creative Commons Lizenz steht und die betreffende Handlung nicht nach gesetzlichen Vorschriften erlaubt ist, ist für die oben aufgeführten Weiterverwendungen des Materials die Einwilligung des jeweiligen Rechteinhabers einzuholen. 